\title{
CONSEQUENCE RELATIONS IN DRT
}

\author{
Seiki Akama \\ Computational Logic Laboratory, Department of Information Systems, \\ Teikyo University of Technology, 2289 Uruido, Ichihara-shi, \\ Chiba, 290-01, Japan. \\ (TEL) +81-436-74-5511, (FAX) +81-436-74-2592. \\ Yotaro Nakayama \\ Nihon Unisys Ltd., Tokyo Bay Development Center, \\ 1-10-9, Shinonome, Koto-ku, Tokyo, 135, Japan.
}

\begin{abstract}
We discuss some consequence relations in DRT useful to discourse semantics. We incorporate some consequence relations into DRT using sequent calculi. We also show some connections of these consequence relations and existing partial logics. Our attempt enables us to display several versions of DRT by employing different consequence relations.
\end{abstract}

\section{Introduction}

Discourse Representation Theory (DRT) due to Kamp (1981) provides a promising framework for discourse semantics. DRT is in fact successul in formalizing several challenging topics, e.g. anaphora, tense and belief; see Asher (1986, 1993) and Heim (1982). Due to its clear semantics with the construction algorithm, DRT is also used as a background theory in computational linguistics; see Wada and Asher (1986) and Ishikawa and Akama (1992).

However, JDRT lacks a deductive (proof theoretic) formulation to serve as an abstract interpreter for discourse understanding, since it is formalized by means of the notion of partial models. This prevents us from ntilizing DRT in varions ways for natural language understanding systems. To make DRT more flexible for computational linguistics, we need to generalize a formal basis in a proof-theoretic fashion. If this is successful, computational lin- guists will be able to reconstruct DRT for their own systems using programming languages like Prolog and LISP. There are perhaps several ways to give an operational semantics of DRT. One of the attractive approaches is to investigate consequence relations associated with $\mathrm{DR} T$. It is thus very useful to study some consequence relations in DRT to develop different versions of DRT.

The purpose of this paper is to explore consequence relations in DRT, one of which exactly produces Kamp's original semantics. We incorporate some consequence relations defined by partial semantics into DRT using sequent calculi. Our attempt enables us to display several versions of DRT by employing different consequence relations. We also show some connections of the proposed consequence relations and partial logics in the literature.

\section{Overview of DRT}

In this section, we give a brief introduction to DRT. For a detailed exposition, the reader should consult Asher (1993). The basic idea of DRT is to formalize a dynamic representation of partial interpretations by means of classical models using a construction algorithm of discourse representation structures (DRSs). Observe that DRSs can be regarded as such abstract objects as partial models, mental representations, or (partial) possible worlds. But, 
such identifications do not seem essential to the significance of $\mathrm{DRT}$.

The language of $\mathrm{DRT}$ is called Discourse Representation Language (DRL), which is like a standard quantifier-free first-order language except discourse referents and conditions. The logical symbols of DRL include $=$ (identity), $\Rightarrow$ (conditional), $\vee$ (disjunction) and $\sim$ (negation). A discourse representation (DR) $\mathrm{K}$ is expressed as a pair $\left\langle U_{K}\right.$, Con $\left.K\right\rangle$, where $U_{K}$ is a set of discourse referents, and Con $K_{K}$ is a set of conditions. Each condition is either atomic or complex. Complex conditions are of the form: $\mathrm{K}_{1} \Rightarrow \mathrm{K}_{2}, \mathrm{~K}_{1} \vee \mathrm{K}_{2}$ or $\sim \mathrm{K}_{1}$, where both $\mathrm{K}_{1}$ and $K_{2}$ are DRs.

A discourse representation structure (DRS) is a partially ordered set of DRs, which can be constructed by means of DRS construction rules whose application reflects the syntactic composition of the sentences in the discourse. When each DR of a DRS is maximal, the DRS is called a complete DRS. Intuitively speaking, each stage in the construction algorithm can be vicwed as a partial possible worlds, in which more information resulting from the processing of a further bit of the discourse changes it into a more precise description of the world.

A model for DRL is an ordered pair $\left(\mathrm{D}_{M}\right.$, $\left.F_{M}\right\rangle$, where $D_{M}$ is the domain of $M$ and $F_{M}$ is an interpretation function of constants and predicates. An embedding function for a DR $K$ in a model $M$ is a mapping from discourse referents in $U_{K}$ into the domain of $M$. An $e x$ tension of an embedding function $f$ for $K$ in $M$ to an embedding function $g$ for $K^{\prime}$ in $\mathrm{M}$ is defined as $g:\left(\operatorname{Dom}(f) \cup U_{K^{\prime}}\right) \rightarrow D_{M}$. We write f. $\subseteq_{K} \mathrm{~g}$ to mean that $\mathrm{g}$ extends an embedding function $f$ to an embedding of $K$ '. 'The notaion $\mathrm{M} \vDash f_{f, K} \mathrm{C}$ abbreviates that $\mathrm{M}$ satisfies $\mathrm{C}$ under $\mathrm{f}$ for $\mathrm{K}$. A proper embedding of $\mathrm{K}$ in $\mathrm{M}$ is an embedding function such that $\mathrm{f} C_{K} \mathrm{~g}$ and for any condition $\mathrm{C}$ in $\mathrm{K}, \mathrm{M} \models_{g, K} \mathrm{C}$. The notions of proper embedding and satisfaction can be extended to general cases by simultaneous recursion; see Asher (1993). A DR $K$ is shown to be true in a model $M$ iff there is a proper embedding of $\mathrm{K}$ in M. A DR K implies a DR K' iff every model in which $\mathrm{K}$ is true is also a model in which $K$ ' is true. 'This definition induces a consequence relation in DRT, but we have no reason to consider it as the only plansible for DRT. In fact, it is our job in this paper to seck alternate definitions.

\section{Consequence Relntions and Sequent Calculi}

A partial semantics for classical logic is implicit in the so-called Beth tableaux. This insight can be generalized to study consequence relations in terms of Gentzen calculi. The first important work in this direction has been done by van Benthem $(1986,1988)$. We here try to apply this technique to DRT. Since our approach can replace the base logic of DRT by other interesting logics, we obtain alternative versions of DRT.

Recall the basic tenet of Beth tableaux. Namely, Beth tableaux (also semantic tableaux) prove $X \rightarrow Y$ by constructing a counterexample of $X \& \sim Y$. In fact, Beth tableaux induce partial semantics in the sense that there may be counterexamples even if a branch remains open. Let $X$ and $Y$ be sets of formulas, and $A$ and $B$ be formulas. And we write $X+Y$ to mean that $Y$ is provable from $X$. Van Benthem's partial semantics for classical logic can be axiomatized by the Gentzen calculıs, which has the axiom of the form:

$$
X, A \vdash A, Y
$$

and the following sequent rules:

$$
\begin{aligned}
& \text { (Weakening) } \mathrm{X} \vdash \mathrm{Y} \Rightarrow \mathrm{X}, \mathrm{A} \vdash \mathrm{A}, \mathrm{Y} . \\
& \text { (Cit) } \mathrm{X}, \mathrm{A} \vdash \mathrm{Y} \text { and } \mathrm{X} \vdash \mathrm{A}, \mathrm{Y} \\
& \quad \Rightarrow \mathrm{X} \vdash \mathrm{Y} . \\
& (\sim \mathrm{R}) \mathrm{X}, \mathrm{A} \vdash \mathrm{Y} \Rightarrow \mathrm{X} \vdash \sim \mathrm{A}, \mathrm{Y} . \\
& (\sim \mathrm{L}) \mathrm{X} \vdash \mathrm{A}, \mathrm{Y} \Rightarrow \mathrm{X}, \sim \mathrm{A} \vdash \mathrm{Y} . \\
& (\& \mathrm{R}) \mathrm{X} \vdash \mathrm{Y}, \mathrm{A} \text { and } \mathrm{X} \vdash \mathrm{Y}, \mathrm{B} \\
& =\Rightarrow \mathrm{X} \vdash \mathrm{Y}, \mathrm{A} \& \mathrm{~B} . \\
& (\& \mathrm{I}) \mathrm{X}, \mathrm{A}, \mathrm{B} \vdash \mathrm{Y} \Rightarrow \mathrm{X}, \mathrm{A} \& \mathrm{~B} \vdash \mathrm{Y} . \\
& (\mathrm{R}) \mathrm{X} \vdash \mathrm{A}, \mathrm{B}, \mathrm{Y} \Rightarrow \mathrm{X} \vdash \mathrm{A} \vee \mathrm{B}, \mathrm{Y} . \\
& (\mathrm{VL}) \mathrm{X}, \mathrm{A} \vdash \mathrm{Y} \text { and } \mathrm{X}, \mathrm{B} \vdash \mathrm{Y} \\
& \Rightarrow \mathrm{X}, \mathrm{A} \vee \mathrm{B} \vdash \mathrm{Y} .
\end{aligned}
$$

Van Benthem's formulation can be extended for partial logics. Because such an extension. 
uses the notion of partial valuations, it is not difficult to recast the treatment for DRT.

Let $\mathrm{V}$ be a partial valuation assigning 0,1 to some atomic formula $p$. Now, we set $V(p)$ $=1$ for $\mathrm{p}$ on the left-hand side and $\mathrm{V}(\mathrm{p})=0$ for $p$ on the right-hand side in an open branch of Beth tableaux. This construction can be easily accommodated to sequent calculi. Then, we can define the following two consequence relations:

(C1) for all $\mathrm{V}$, if $\mathrm{V}($ Pre $)=1$

$$
\text { then } V(\text { Cons })=1 \text {, }
$$

(C2) for all $V$, if $V$ (Pre) $=1$

$$
\text { then } V \text { (Cons) } \neq 0 \text {, }
$$

where Pre and Cons stand for premises (antecedent) and conclusion (succedent) of a sequent, respectively. In a classical setting, (C1) and (C2) coincide. It is not, however, the case for partial logics.

The Gentzen calculus G1 for $\mathrm{C1}$ is obtainable from the above system without right $(\sim)$ rule by introducing the following rules:

$$
\begin{gathered}
(\sim \sim \mathrm{R}) \mathrm{X} \vdash \mathrm{A}, \mathrm{Y} \Rightarrow \mathrm{X} \vdash \sim \sim \mathrm{A}, \mathrm{Y} . \\
(\sim \sim \mathrm{L}) \mathrm{X}, \mathrm{A} \vdash \mathrm{Y} \Rightarrow \mathrm{X}, \sim \sim \mathrm{A} \vdash \mathrm{Y} . \\
(\sim \& \mathrm{R}) \mathrm{X} \vdash \sim \mathrm{A}, \sim \mathrm{B}, \mathrm{Y} \\
\quad \Rightarrow \mathrm{X} \vdash \sim(\mathrm{A} \& \mathrm{~B}), \mathrm{Y} . \\
(\sim \& \mathrm{~L}) \mathrm{X}, \sim \mathrm{A} \vdash \mathrm{Y} \text { and } \mathrm{X}, \sim \mathrm{B} \vdash \mathrm{Y} \\
\Rightarrow \mathrm{X}, \sim(\mathrm{A} \& \mathrm{~B}) \vdash \mathrm{Y} . \\
(\sim \vee \mathrm{R}) \mathrm{X} \vdash \sim \mathrm{A}, \mathrm{Y} \text { and } \mathrm{X} \vdash \sim \mathrm{B}, \mathrm{Y} \\
\Rightarrow \mathrm{X} \vdash \sim(\mathrm{A} \vee \mathrm{B}), \mathrm{Y} . \\
(\sim \vee \mathrm{L}) \mathrm{X}, \sim \mathrm{A}, \sim \mathrm{B} \vdash \mathrm{Y} \\
\Rightarrow \mathrm{X}, \sim(\mathrm{A} \vee \mathrm{B}) \vdash \mathrm{Y} .
\end{gathered}
$$

Van Benthem (1986) showed that G1 is a Gentzen type axiomatization of $\mathrm{C} 1$. To guarantee a cutfree formulation, we need to modify van Benthem's original system. We denote by GC1 the sequent calculus for $\mathrm{GC} 1$, which contains the axioms of the form: (A1) $A \vdash A$ and (A2) A, $\sim \mathrm{A} \vdash$, with the right and left rules for $(\&)$, ( $\vee),(\sim \sim),(\sim \&)$ and $(\sim \vee)$ together with (Weakening) and (Cut). It is shown that GC1 is equivalent to $G 1$ withont any difficulty. As a consequence, we have:

\section{Theorem 1}

C1 can be axiomatized by GC1.

The Gentzen system GC2 for $\mathrm{C} 2$ can be obtained from (GC1) by adding the next axiorn:
(A3) $\vdash \mathrm{A}, \sim \mathrm{A}$.

\section{Theorem 2}

C2 can be axionatized by GC2.

There are alternative ways to define consequence relations by means of sequent calculi. For example, it is possible to give the following alternate definitions.

(C3) for all $V$, if $V($ Pre $)=1$

$$
\begin{aligned}
& \text { then } V(\text { Cons })=1 \\
& \text { and if } V(\text { Cons })=0 \\
& \text { then } V(\text { Pre })=0 .
\end{aligned}
$$

The new definition obviously induces inconsistent valuations. The Gentzen system $\mathrm{GC3}$ is obtainable from GC1 by replacing (A2) by the following new axiom:

$$
\text { (A4) A, } \sim \mathrm{A} \vdash \mathrm{B}, \sim \mathrm{B} .
$$

\section{Theorem 3}

C3 can be axiomatized by GC3.

\section{Relation to Partial Logies}

In this section, we compare the proposed Gentzen systems with some existing partial logics, in particular, three-valued and four-valued logics in the literature; see Urquhart (1986). To make connections to partial logics clear, we extend DRL with weak negation " $\neg$ " to express the lack of truth rather than verification of falsity in discourses. We denote the extended language by EDRL. In the presence of two kinds of negation, we can also define two kinds of implication as material implications. We need the next rules for weak negation:

$$
(\neg R) X, A \vdash Y \Rightarrow X \vdash \neg A, Y \text {. }
$$$$
(\neg L) X \vdash A, Y \Longrightarrow X, \neg A \vdash Y \text {. }
$$

In fact, these rules provide a new consequence reation of EDRL denoted by $\models_{E D R L}$. Our first result is concerned with the relationship of GC1 and Kleene's (1952) strong three-valued logic KL, namely

\section{Theorem 4}

The consequence relations of GC1 and $\mathrm{KL}$ are equivalent.

From this theorem, EDRL can be identified with the extended Kleene logic EKL. Let A $\rightarrow w_{w} \mathrm{~B}$ be an abbreviation of $\neg \mathrm{A} \vee \mathrm{B}$. Then, we can also interpret Lukasiewicz's three-valued 
$\operatorname{logic} \mathrm{L}_{3}$. In fact, the Lukasiewicz implication $\supset$ can be defined as follows:

$$
\mathrm{A} \supset \mathrm{B}=\operatorname{def}(\mathrm{A} \rightarrow w \mathrm{~B}) \&(\sim \mathrm{B} \rightarrow w \sim \mathrm{A})
$$

which implies

$$
\sim \mathrm{A} \text {. }
$$$$
\models_{E K L} \mathrm{~A} \supset \mathrm{B} \text { iff } \mathrm{A} \models_{E K L} \mathrm{~B} \text { and } \sim \mathrm{B} \models_{E K L}
$$

This is closely related to the consequence relation $\mathrm{C} 3$.

\section{Theorem 5}

$\mathrm{A} \vdash_{C 3} \mathrm{~B}$ iff $\vdash_{E K L} \mathrm{~A} \supset \mathrm{B}$.

If we drop ( $A 2$ ) from $\mathrm{GC1}$, we have the sequent calculus $\mathrm{GC1}^{-}$, which is shown to be equivalent to Belnap's (1977) four-valued logic BEL.

\section{Theorem 6}

$\vdash_{B E L}=\vdash_{G C 1^{-}}$.

The four-valued logic BEL can handle both incomplete and inconsistent information. We believe that four-valued semantics is plausible as a basis for representational semantics like DRT, which should torelate inconsistent information in discourses. In view of these results, we can develop some versions of DRT which may correspond to current three-valued and four-valued logics; see Akama (1994).

\section{Conclusions}

We have studied a proof-theoretic foundation for DRT based on consequence relations defined by partial semantics. These consequence relations yield alternative versions of DRT to be used for different applications. We have noted some connections between these relations and partial logics, in particular three-valued and four-valued logics. We believe that the significance of our work lies in reformulating DRT in sequent calculi to be easily applied to computational linguistics.

There are several topics that can be further developed. First, we should give a more detailed discussion of what sort of completeness proof is involved, although we have established some correspondence results. Second, it is very interesting to show how the proposed consequence relations affect DRT in more detailed ways. Third, we need to extend the present work for the predicate case to take care to cap- ture the dynamic effect of the quantificational structure of DRT.

\section{References}

Akama, S. (1994): A proof system for useful three-valued logics, to appear in Proc. of Japan-CIS Symposium on Knowledge Based Software Engineering.

Asher, N. (1986): Belief in discourse representation theory, Journal of Philosophical Logic 15, 127-189.

Asher, N. (1993): Reference to Abstract Objects in Discourse, Kluwer, Dordrecht.

Belnap, N. D. (1977): A useful four-valued logic, J. M. Dunn and G. Epstein (eds.), Modern Uses of Multiple-Valued Logic, 8-37, Reidel, Dordrecht.

Heim, I. (1982): The Semantics of Indefinite and Definite Noun Phrases, Ph. D. dissertation, University of Massachussetts at Amherst.

Ishikawa, A. and A kama, S. (1991): A semantic interface for logic grammars and its application to DRT, C. Brown and G. Koch (eds.), Natural Language Understanding and Logic Programming III, 281292, North-Holland, Amsterdam.

Kamp, H. (1981): A theory of truth and semantic representation, J. Groenendijk, T. Janssen and M. Stokhof (eds.), Formal Methods in the Study of Language, 277-322, Mathematisch Centrum Tracts, Amsterdam.

Kleene, S. C. (1952): Introduction to Metamathematics, North-Holland, Amsterdam.

Urquhart. A. (1986): Many-valued logic, 1). Caldbay and fi. Gineuthuer (eds.). Handbook a) Phalosophlacal hogic vol. IV. i1-116. Riciclel, Dordroctal.

Wada, H. and Asher, N. (1986): BUILDRS: An implementation of DR Theory and LFG, Proc. of COLING-86, 540-545. 